\title{
In Memoriam : Serge Villeret (1911-1994)
}

Monsieur S. Villeret nous a quitté le 13 octobre 1994 à l'âge de 83 ans. Témoin et acteur de la rapide transformation de l'université française lors des cinquante dernières années, il fut également le digne représentant d'une certaine démocratisation de la société française par l'enseignement public. En effet, tout en étant instituteur (1930-1942), il eut la possibilité de continuer ses études et obtenir une Licence de Sciences Naturelles. Intégré à l'université en 1942 comme assistant, ses recherches s'orientèrent vers l'écologie des algues d'eau douce. En France, il fut un des précurseurs de cette approche en essayant de faire la liaison entre les paramètres physicochimiques et les groupements d'algues. Ses recherches lui permirent, en $19 ! 3 ;: z: j$ : tenir une Thèse de Doctorat d'Etat intitulée « Contribution à la biologie des algues des tourbières à sphaignes ». Ses différents travaux ont également permis de faire connaître plus de 800 espèces de la région bretonne ; par ailleurs, avec l'aide de ses élèves, il s'est également intéressé à l'inventaire de la flore algale des milieux stagnants de la Double (Dordogne) et des Pyrénées.

Nommé professeur en 1956, puis professeur titulaire de la chaîne de physiologie végétale nouvellement créée à Rennes (1958), il a alors orienté une grande partie de ses recherches vers la physiologie végétale des algues d'eau douce. Il a notamment montré que certaines algues pouvaient utiliser des uréides glyoxyliques. Ses différents travaux ont fait l'objet d'une cinquantaine de publications et de nombreuses communications à des congrès nationaux et internationaux.
En tant que professeur à l'Université de Rennes, Monsieur Villeret assura le développement de l'enseignement de la physiologie végétale et contribua fortement à la mise en place d'en enseignement d'hydrobiologie.

Parallèlement à son travail de recherche et d'enseignement, Monsieur Villeret eut de multiples activités. Il fait partie de ces enseignants qui ont assuré le passage d'une université réservée à quelques-uns à l'enseignement de masse nécessité par les besoins de la société. Il a participé très activement à cette mutation en acceptant des charges administratives : Assesseur du Doyen et Directeur de l'Unité de Recherche et d'Enseignement du Comportement et de l'Environnement. Il s'est également investi dans d'autres tâches collectives plus obscures telles l'animation de la Société Scientifique de Bretagne et de son service d'échanges internationaux. Il fut également un des membres fondateurs de l'Association Française de Limnologie.

Monsieur Villeret ne se contentait pas d'être un scientifique bien au chaud dans sa tour d'ivoire d'universitaire ; il s'intéressait notamment aux pays en voie de développement ; c'est ainsi qu'il assura pendant plusieurs années l'enseignement de la physiologie végétale à la toute nouvelle université du Niger.

A ses étudiants, Monsieur Villeret laisse le souvenir d'un enseignant qui savait rendre digeste même le cycle de Krebs, aux membres de son laboratoire, celui d'un chercheur très critique mais jamais à court d'idées et à la communauté scientifique, celui d'un homme jovial, sachant plaisanter et facile à vivre.

R. Le Cohu . 


\section{Principaux travaux de Monsieur Villeret portant sur les algues d'eau douce}

1. - Villeret S. 1938. - La végétation de la lande d'Ouée. Bull. Soc. Sci. Bretagne, 15 : 206-227.

2. - Villeret S. 1948. - La kéritomie et la fragmentation du thalle chez l'Oscillaire Oscillatoria borneti Zukal. Bull. Soc. Sci. Bretagne, 23 : 85-92.

3. - Villeret S. 1948. - Contribution à la connaissance de la flore algologique des environs de Rennes. Bull. Soc. Sci. Bretagne, 23 : 85-92.

4. - Villeret S. 1951. - Quelques espèces d'algues d'eau douce nouvelles. Bull. Soc. Bot. Fr., 98 : 37-40.

5. - Villeret S. 1951 a. - Recherches sur le rôle du $\mathrm{CO}_{2}$ dans l'acidité des eaux des tourbières à sphaignes. C.R. Acad. Sci. Paris, 232 : 1583-1585.

6. - Villeret S. 1951 b. - Le déphasage de la morphogénèse et de la division cellulaire chez les Desmidiées unicellulaires. Bull. Soc. Sci. Bretagne, 26 : 49-58.

7. - Villeret S. 1953. - Quelques algues d'eau douce, rares ou nouvelles. Bull. Soc. Bot. Fr., 100 : 71-74.

8. - Villeret S. 1953 a. - Etude hydrobiologique de quelques mares des environs de Rennes. I. Mare des Buttes. Bull. Soc. Sci. Bretagne, 28 : 97-111.

9. - Villeret S. 1953 b. - Etude hydrobiologique de quelques mares des environs de Rennes. Bull. Soc. Sci. Bretagne, 28 : 112-131.

10. - Villeret S. 1954 a. - Contribution à la biologie des algues des tourbières à sphaignes. Thèse Sci. Paris 1953. Bull. Soc. Sci. Bretagne, H. S. 29 : 246 p.

11. - Villeret S. 1954 b. - La végétation algale de la tourbière de Nesnay en Plouneour. Bull. Soc. Sci. Bretagne, $29: 17-31$.

12. - Villeret S. 1958. - Recherches hydrobiologiques sur les étangs calcaires de la Haute-Sève (Ille et Vilaine). Bull. Soc. Sci. Bretagne, 33 : 65-69.
13. - Verger-Lagadec F. \& Villeret S. 1963. - Les algues d'eau douce du Massif du Néouvielle (HautesPyrénées). Bull. Soc. Hist. Nat. Toulouse, 98 : 501-519.

14. - Savouré B. \& Villeret S. 1964. - Contribution à l'étude hydrobiologique de la Bretagne. I. Florule algale et typologie des eaux de quelques étangs de l'Ille et Vilaine (à l'exception des diatomées). Bull. Soc. Sci. Bretagne, 39 : 233-241.

15. - Villeret S. 1965 a. - Nutrition azotée des algues d'eau douce. Utilisation des composés nitroaryl. Bull. Soc. Fr. de Physiologie Végétale, 11: 282-287.

16. - Villeret S. 1965 b. - Quelques problèmes d'écophysiologie des végétaux aquatiques (Algues). La sexualité. Bull. Soc. Fr. de Physiologie Végétale, $11: 287-298$.

17. - Villeret S., Citharel L. \& Verger-Lagadec F. 1972. - Contribution à l'étude hydrobiologique des étangs de la Double (Dordogne). Bull. Soc. Sci. Bretagne, 47 : 97-117.

18. - Villeret S., Bertru G., Le Cohu R. \& Lenoir M. 1972. - Recherches hydrobiologiques sur les étangs et les lacs de la région du Carlite (P.O.). II. Les populations algales. $1^{\text {ere }}$ partie : la florule diatomique. Bull. Soc. Sci. Bretagne, 47 : 193-208.

19. - Villeret S., Bertru G., Le Cohu R. \& Lenoir M. 1973. - Recherches hydrobiologiques sur les étangs et les lacs de la région du Carlite (P.O.). II. Les populations algales. $2^{\mathrm{e}}$ partie : les algues autres que les Bacillariophycées. Bull. Soc. Sci. Bretagne, $48:$ 217-227.

20. - Villeret S. 1973. - Recherches sur le métabolisme azoté des Zygnématales. Utilisation de l'urée et des nitrates par l'algue Cosmarium formosulum Hoff (Desmidiacée - Zygnématales). Verh. Internat. Verein. Limnol., 18 : 1375-1379. 